\title{
Optimal Technology
}

\section{Nader Barsoum*}

Department of Electrical and Electronic Engineering, University Malaysia Sabah, Malaysia

It is my pleasure to introduce the Global Journal of Technology and Optimization GJTO which successfully started the first volume in 2010 to publish high quality peer reviewed papers online. The plan was one volume per year on the new technology developments that required investigation and more researches to obtain the optimal design and operation to provide high quality features with the lowest cost effectives.

The journal starts to publish the research papers in 2013 with OMICS-online and encourages research motivated by the challenging problems related to new technology that document the development and use of industrial and systems engineering tools and techniques including operations research, statistics, information systems, work measurement, and human factors applications.

High quality submissions that advance the research and that contribute something new to the literature on New Technology are encouraged. The special focus of the GJTO forum is to publish groundbreaking applications and applied research results.

The target audience of the journal will be composed of professionals and researchers working in the fields of technology and optimization in various disciplines. Unpublished papers and extended versions of papers presented at conferences can be submitted for possible publication in the journal. Technical papers, white papers and experience reports are also welcome for evaluation in GJTO.

The platform is the aim for all researchers, engineers, practitioners, academicians, students and industrial professionals publish their research results and development activities in the area of technology and its optimization techniques. Researchers will give the optimum materials in these areas since many engineers, scientists, finance, business and economists suffer from a problem of developing a system that can cope with variations of system or control parameters, measurements uncertainty and complex multi-objective optimization criterion. The need for a prior knowledge and the inability to learn from past experience make the design of robust, adaptive and stable systems a difficult task.

Currently, researches on energy resources, energy saving, energy planning and the global warming are found to be a great importance for future alternative replacement of fossil fuel. The new technology on smart power grid and micro grid and the use of micro-controller programming are also important.

Computational Intelligence has been proven to have successful solution of complex optimization problems by fuzzy logic, neural Network, Evolutionary Algorithms, Genetic with line or pattern search, Particle Swam Optimization, and Hybrid System Optimization in variety of engineering, science, business, finance, economics, management and hybrid energy resources applications. They include system identification, parameter estimation, multi-objective optimization, robust solution, adaptive system, self organization and failure analysis. Control systems and stability are also employed specially the treatments of disable patients by robotics. The networking and wireless sensors and communication with mobile are the most important technology these days; in particular the measurements of transmitted signals of human body that related to blood test can now be found in mobile android phone. Also, controlling the objects by mobile or Ethernets is of important task. Software Engineering and the new design of optimal mechanical systems are also employed in this journal. The goal is to bring together researchers working on the development of techniques and methodologies to improve the performance of power systems, energy planning and environments, controllers and robotics, operation research, and modern artificial computational intelligent techniques.

Hope that this journal that published by OMICS-online will provide inspiring future technology with an optimal states.
*Corresponding author: Nader Barsoum, Professor, Department of Electrical and Electronic Engineering, University Malaysia Sabah, Malaysia, Tel: +60163541546. E-mail: pcoglobal@gmail.com

Received August 19, 2013; Accepted August 20, 2013; Published August 25, 2013

Citation: Barsoum N (2013) Optimal Technology. Global J Technol Optim 4: e101. doi: 10.4172/1410-3217.1000e101

Copyright: (c) 2013 Barsoum N. This is an open-access article distributed under the terms of the Creative Commons Attribution License, which permits unrestricted use, distribution, and reproduction in any medium, provided the original author and source are credited. 